\title{
Infarto cerebral hemisférico: algoritmo de tratamento baseado em evidência
}

\author{
Wuilker Knoner Campos ${ }^{1}$, José Antônio Damian Guasti²
}

\section{RESUMO}

O infarto cerebral hemisférico maligno tem mortalidade de $80 \%$, apesar do tratamento conservador. Ele representa infarto de mais de $50 \%$ do território da artéria cerebral média (ACM), entretanto, nem todos os pacientes irão desenvolver o curso maligno. O objetivo desta revisão foi encontrar os fatores preditivos do curso maligno do edema cerebral maligno e, a partir desses fatores, propor um algoritmo de tratamento e tomadas de decisões para o infarto cerebral hemisférico. Os preditores foram pesquisados em artigos no PubMed. Com base nos preditores encontrados com valor estatístico significativo, estruturou-se um algoritmo de gerenciamento para o infarto cerebral hemisférico. Os fatores preditivos de curso maligno foram: NIHSS > 15 hemisfério dominante e $>20$ não dominante; oclusão da artéria carótida interna ( $\mathrm{ACl}$ ) ipsilateral à malformação do círculo de Willis; circulação colateral deficiente; tomografia computadorizada (TC) hipodensidade $>50 \%$ e ressonância magnética (RM) difusão $>145 \mathrm{~cm}^{3}$ do território da ACM; outro território vascular envolvido; perda da autorregulação; potencial evocado auditivo patológico; pressão intracraniana $(P I C)>35 \mathrm{mmHg}$ e valores de pico de aminoácidos excitatórios medidos na microdiálise. Entretanto, esses dois últimos fatores aparecem apenas depois que o paciente está herniado. Os trials europeus definiram nível de evidência 1 para craniectomia descompressiva. Craniectomia descompressiva é o tratamento de primeira escolha para os pacientes $<60$ anos com curso maligno do infarto hemisférico $<48 \mathrm{~h}$. Os fatores preditivos são importantes ferramentas para tomada de decisão quanto à indicação cirúrgica precoce.

\section{PALAVRAS-CHAVE}

Craniectomia descompressiva, infarto cerebral, acidente cerebral vascular/cirurgia, infarto da artéria cerebral média.

\section{ABSTRACT}

Hemispheric ischemic stroke: evidence-based management algorithm

Massive hemispheric infarctions (MHI) constitute $10 \%$ of all ischemic strokes and have a mortality rate of $80 \%$, under conservative treatment. This stroke presents a hypodensity covering more than $50 \%$ of the middle cerebral artery (MCA) territory, however, not all the patients develop the malignant course of hemispheric infarctions. The goal of this study was to determine the predictors of fatal brain edema and to propose an evidence-based management algorithm. Search for predictors of MHI with malignant edema was performed in PubMed and Cochrane data base. An evidence-based management algorithm was structured from this study. Different predictors of fatal brain edema formation have been identified: NIHSS > 15 dominant and > 20 non dominant hemisphere; carotid occlusion with abnormal ipsilateral circle of Willis; collateral deficit; early computed tomography (CT) hypodensity involving $>50 \%$ or DWI $>145 \mathrm{~cm}^{3}$ of the MCA territory; involvement of additional vascular territories; impaired cerebral autoregulation; pathological auditory potentials evoked; ICP > $35 \mathrm{mmHg}$; microdialysis with peak values of the excitatory amino acids. Decompressive hemicraniectomy is the first choice to treatment for MHI with malignant brain edema in patients $>60$ years and $<48$ hours after stroke. To know predictors with evidence level are important tools to make decision about surgical indication.

\section{KEYWORDS}

Decompressive craniectomy, cerebral infarction, accidente cerebrovascular/surgery, infarction, middle cerebral artery.

1. Neurocirurgião do Instituto de Neurocirurgia Neuron, Florianópolis, SC. Médico pós-graduado em Neurointensivismo pelo Hospital Sírio-Libanês.

2. Chefe do serviço de Neurocirurgia do Hospital Federal Bonsucesso, Rio de Janeiro, RJ.

Trabalho apresentado pelo autor como tese de conclusão do Curso de Pós-graduação lato sensu em Neurointensivismo, Instituto de Ensino e Pesquisa do Hospital Sírio-Libanês, São Paulo, SP. 


\section{Introdução}

Acidente vascular encefálico (AVE) é a segunda causa geral de morte no mundo, e até $10 \%$ dos pacientes com essa patologia desenvolverão infarto cerebral hemisférico, que segue um curso maligno na maioria dos casos, determinando a morte do paciente em $80 \%$, apesar do tratamento clínico. ${ }^{4,34} \mathrm{O}$ termo "infarto cerebral hemisférico maligno" foi introduzido por Hacke et al. ${ }^{14}$ em 1996 e atualmente é definido como infarto de mais de $50 \%$ do território da artéria cerebral média (ACM) visto na tomografia computadorizada (TC) e/ ou ressonância magnética $(\mathrm{RM})$ de crânio consoantes com apresentação clínica (Figura 1)..$^{30,44,49}$

Os pacientes que desenvolvem o curso maligno do infarto cerebral hemisférico por causa do edema cerebral tipicamente deterioram dentro das primeiras 48 horas, apresentando um pico máximo do edema entre 48-96 horas. ${ }^{12}$ Dessa forma, a morte encefálica por causas neurológicas ocorre dentro da primeira semana; após esse período crítico, a causa da morte passa a ser por condições clínicas associadas, como pneumonia e tromboembolismo pulmonar.

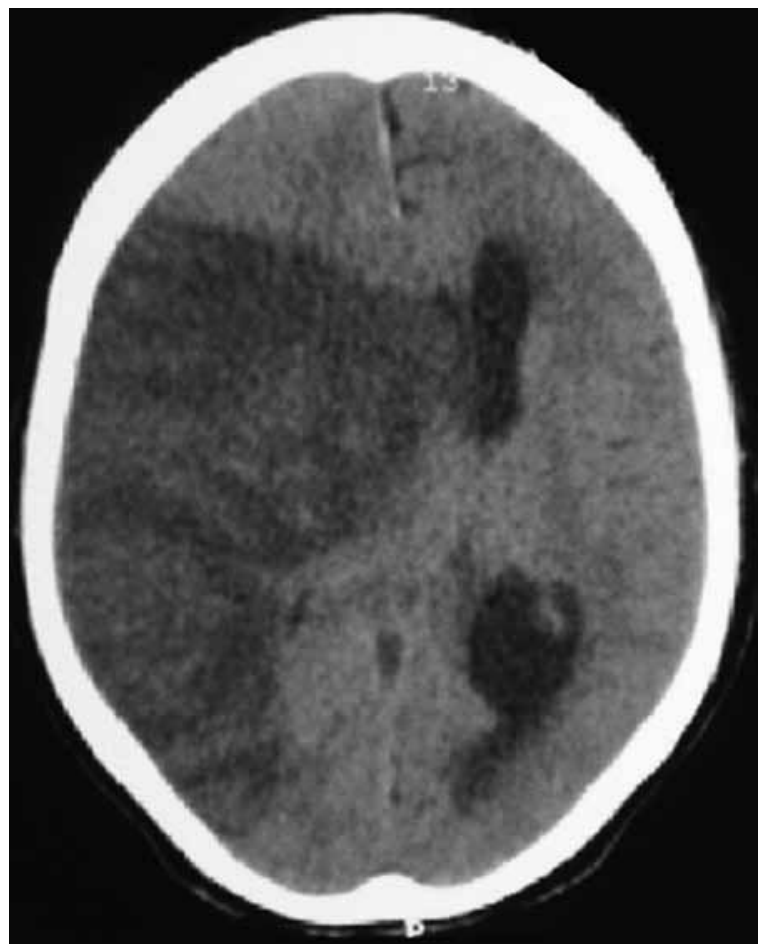

Figura 1 - Tomografia computadorizada axial de crânio: paciente feminino, 45 anos, pós-operatório de cirurgia cardíaca (troca de válvula aórtica) com infarto de mais de $50 \%$ da artéria cerebral média direita. Nota-se o efeito de massa do infarto com desvio de linha média de mais de $10 \mathrm{~mm}$ contralateral ao infarto, ventrículo lateral direito rechaçado e apagamento das cisternas, sulcos e giros. Após 32 horas do ictus, realizou craniectomia descompressiva com resultado satisfatório (mRS 3 na alta).
A partir da segunda metade do século passado iniciou-se um debate sobre qual a melhor opção de tratamento para esses pacientes, haja vista o curso catastrófico da doença. O tratamento medicamentoso, que falhou em mostrar seu benefício, foi perdendo espaço para a craniectomia descompressiva, mostrando que, além de reduzir significativamente a mortalidade (de $78 \%$ para $29 \%$ ), melhora a condição funcional dos pacientes sobreviventes com nível de evidência significativa. ${ }^{16,20,46}$

Entretanto, nem todos os pacientes com AVE hemisférico irão se beneficiar da craniectomia descompressiva em decorrência de sua condição clínica, idade ou prognóstico. Dessa forma, uma indicação desnecessária desse procedimento poderia não só acrescentar riscos ao paciente como também prolongar o sofrimento deste. $\mathrm{O}$ tratamento nunca pode ser pior do que a história natural da doença. Dessa forma, é preciso estandardizar critérios para a indicação dessa cirurgia. O objetivo desta revisão de literatura foi rever as atuais evidências sobre as diversas opções de tratamento para o infarto cerebral hemisférico, identificar os fatores preditivos do seu curso maligno e, com base nesses dados, propor um algoritmo de tratamento e tomadas de decisões para o infarto cerebral hemisférico.

\section{Materiais e métodos}

Os artigos foram identificados de 1935 a 2009 a partir da base de dados PubMed, usando as palavras-chave decompressive surgery; massive hemispheric infarctions; malignant MCA infarction; hemicraniectomy; MCA occlusion. A partir dos artigos encontrados, rastrearam-se ainda as referências bibliográficas destes. Conforme o nível de evidência do U.S. Preventive Services Task Force, os fatores preditivos significantes encontrados (Quadro 1) foram utilizados para estruturar um algoritmo de tratamento e tomadas de decisões para o infarto cerebral hemisférico (Figura 2).

\section{Preditores do AVE maligno}

Em um estudo pos mortem ${ }^{36} \mathrm{com} 353$ pacientes com AVE supratentorial, foram encontrados 45 pacientes (13\%) com AVE isquêmico hemisférico e, destes, cerca de 35 pacientes ( $75 \%$ dos 45 pacientes) morreram na primeira semana, sendo a maioria nos primeiros quatro dias com a presença de herniação encefálica ( $88 \%$ herniação transtentorial, $34 \%$ subfalcinea e $83 \%$ foraminal). 
Quadro 1 - Fatores preditivos relacionados ao curso maligno do edema cerebral no infarto cerebral hemisférico, segundo a significância estatística encontrada

Fatores significantes $(\mathrm{P}<0,05)$

- $\quad$ NIHSS $>15$ hemisfério dominante ou NIHSS $>20$ hemisfério não dominante

- TC de crânio com hipodensidade $>50 \%$ do território da ACM

- $\mathrm{RM}$ difusão $>145 \mathrm{~cm}^{3}$ do território da $\mathrm{ACM}$

- Outro território vascular cerebral acometido (ACA, ACP, A. coroideia)

- Oclusão tipo I da $\mathrm{ACM}^{*}$

- Oclusão da ACI ipsilateral a uma malformação do círculo de Willis

- Circulação colateral via AcomA (regra dos 5 segundos) ruim

- Perda da autorregulação cerebral

- BAEP patológico

- $\quad \mathrm{PIC}>35 \mathrm{mmHg}$

- Microdiálise com aminoácidos excitatórios em valores de pico
Fatores não significantes

- Idade, sexo, raça

- Febre, frequência cardíaca

- Hemisfério acometido

- Cefaleia, náuseas/vômitos precoce

- Hipertensão arterial, cardiopatia, diabetes, fibrilação atrial

- História de IAM, AVE ou AIT, tabagismo

- Glicemia, $\mathrm{Na}, \mathrm{K}, \mathrm{Mg}$, hemograma, colesterol

- Glasgow

- Intervalo QT

- PET e SPECT

- Outros

ACA: artéria cerebral anterior; ACM: artéria cerebral média; ACP: artéria cerebral posterior; AcomA: artéria comunicante anterior; AIT: acidente isquêmico transitório; AVE: acidente vascular encefálico; BAEP: Brainstem auditory evoked potential; IAM: infarto agudo do miocárdio; NIHSS: National Institute of Health Stroke Scale; PIC: pressão intracraniana. * Oclusão do segmento M1.

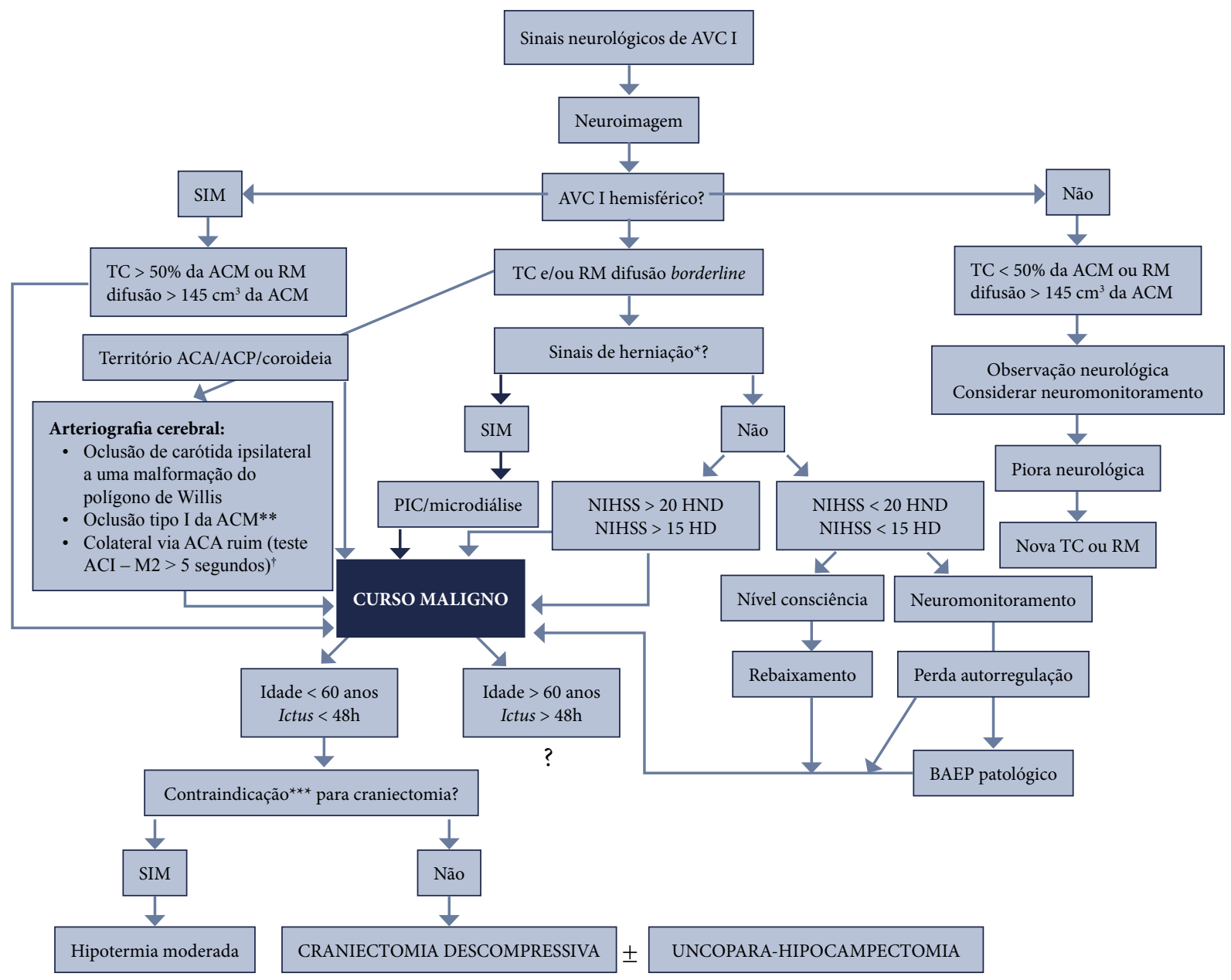

Figura 2 - Algoritmo de tratamento do infarto cerebral hemisférico baseado em evidência.

ACA: artéria cerebral anterior; ACM: artéria cerebral média; ACP: artéria cerebral posterior; AVC I: acidente vascular cerebral isquêmico; BAEP: Brainstem auditory evoked potential; HD: hemisfério dominante; HND: hemisfério não dominante; M2: segundo segmento da ACM; NIHSS: National Institute of Health Stroke Scale; PIC: pressão intracraniana; RM: ressonância magnética; TC: tomografia computadorizada. * Sinais de herniação: anisocoria ou pupilas fixas/dilatadas; decerebração contralateral à hemiplegia; TC com desvio linha média >10 mm. ** Oclusão tipo I da ACM refere-se ao envolvimento do segmento M1 (território vascular profundo).

${ }^{* * *}$ Contraindicações para craniectomia descompressiva: distúrbio coagulação não corrigível; cardiopatia severa; doença terminal; infarto bilateral de ACM ou ACA; dependência funcional prévia. 
Os outros 10 pacientes (25\%) morreram entre a segunda e décima semana de causas clínicas gerais. Entretanto, a necropsia desse segundo grupo de morte tardia também revelou herniação encefálica em proporções semelhantes às do primeiro grupo ( $90 \%$ herniação transtentorial, $30 \%$ subfalcinea e $50 \%$ foraminal). Ambos os grupos apresentaram herniação encefálica praticamente nas mesmas proporções, porém a continuação da necropsia revelou que o primeiro grupo apresentou lesões secundárias de tronco mais frequentes ( $89 \%$ edema e $71 \%$ hemorragia de tronco) que o segundo grupo (50\% edema e $20 \%$ hemorragia) e esse dado foi o diferencial determinante para o curso maligno do infarto cerebral hemisférico dos pacientes herniados.

Dessa forma, entende-se como curso maligno do infarto cerebral hemisférico o paciente que apresentar herniação encefálica associado à lesão secundária de tronco encefálico, e que nem todo paciente com infarto cerebral hemisférico desenvolverá o curso maligno, mostrando que existem algumas variáveis que determinam o curso maligno do edema associado ao infarto cerebral hemisférico. Um grande número de variáveis tem sido estudado na literatura como possíveis preditores do desenvolvimento desse edema.

\section{Dados clínicos demográficos}

Os principais dados clínicos sugeridos na literatura, como idade, sexo, hemisfério, cefaleia, náusea/vômito precoce, hipertensão arterial, cardiopatia, diabetes, glicemia, sódio, potássio, magnésio, frequência cardíaca, Glasgow, intervalo QT, história de AVE, contagem de leucócitos e febre, não tiveram valor preditivo estatístico suficiente para determinar o curso do infarto cerebral. ${ }^{26,8,17,2}$ O NIHSS (National Institute of Health Stroke Scale) $>20$ no hemisfério não dominante e NIHSS > 15 no hemisfério dominante dentro de 48 horas teve correlação com o curso maligno o infarto cerebral na maioria dos trabalhos. ${ }^{26,27,29}$

Jaramillo et al. ${ }^{19}$, em um estudo pos mortem, mostraram que o curso maligno do infarto cerebral esteve mais frequentemente associado com infarto do território superficial e profundo da ACM, assim como quando outros vasos como coroideia e cerebral anterior estavam envolvidos. Além disso, mostraram também que a oclusão da carótida ipsilateral a uma anormalidade do círculo de Willis é preditiva de infarto cerebral maligno.

\section{Neuroimagem}

Muitos preditores radiológicos têm sido sugeridos na literatura, entretanto, o único preditor radiológico confirmado em todos os trabalhos revisados foi a hipo- densidade com extensão de mais de 50\% no território da ACM na TC de crânio (Figura 1). Demonstrou-se que esse critério foi capaz de predizer fatalidade no seguimento em $85 \%$ dos casos com especificidade de $94 \%{ }^{8,13-15,23,26,47}$

Oppenheim et al. ${ }^{37}$, utilizando a RM por difusão, encontraram um volume médio de $244 \mathrm{~cm}^{3}$ nos pacientes com infarto cerebral hemisférico e um ponto de corte de $145 \mathrm{~cm}^{3}$ para predizer o curso do edema cerebral dentro das primeiras 14 horas após o ictus com alta sensibilidade e especificidade, entretanto, nas primeiras horas de infarto essa sensibilidade é mais baixa. Nessa fase ultraprecoce ( $<6$ horas), outras variáveis como arteriografia digital ou angio-RM são preditores mais sensíveis para o infarto cerebral hemisférico. ${ }^{1,35}$

Bozzao et al. ${ }^{5}$, comparando arteriografia precoce com TC após três meses de seguimento em pacientes com AVC isquêmico supratentorial, concluíram que a presença de circulação colateral via artéria comunicante anterior (AComA) reduziu o tamanho final do infarto cerebral em pacientes com oclusão do tronco da ACM e essa circulação colateral foi mais eficiente nos pacientes sem estenose significativa da artéria carótida interna (ACI) extracraniana. Entretanto, apesar de boa circulação colateral, pacientes com oclusão da ACM desenvolveram infarto em território profundo em função de as artérias lenticuloestriadas serem vasos terminais sem possibilidade de anastomose. A circulação colateral pode ser avaliada com base na "regra dos 5 segundos" na arteriografia cerebral como boa, pobre ou ausente. ${ }^{40} \mathrm{~A}$ circulação colateral é considerada boa quando o tempo de enchimento do seguimento da ACI contralateral para M2 ipsilateral for $<5$ segundos, dessa forma o infarto cortical e subcortical não se desenvolve e o infarto fica limitado aos gânglios da base. Por outro lado, se esse tempo for $>5$ segundos, ocorrerá infarto hemisférico. Essa regra foi capaz de predizer a extensão do infarto mesmo antes de a TC demonstrar hipodensidade. Outros trabalhos também encontraram a correlação das colaterais com o curso do infarto cerebral. ${ }^{25}$

A utilização do SPECT para avaliação prognóstica feita por Limburg et al. ${ }^{28}$ demonstrou que o tamanho do déficit de fluxo inicial se relaciona fortemente com o curso do infarto cerebral. Neste trabalho, todos os pacientes que tiveram os maiores déficit de fluxos morreram em seis dias em consequência de herniação cerebral. Outro exame estudado ${ }^{8}$ foi o PET com flumazenil (FMZ-PET), que mostrou uma relação significativa entre a extensão da área infartada e de penumbra nos pacientes com curso maligno. Entretanto, mais estudos prospectivos são necessários para validação desses dois métodos. 


\section{Neuromonitoramento multimodal}

Enquanto os métodos de imagem fornecem uma ideia estática e vertical do processo isquêmico cerebral, a neuromonitorização com microdiálise, pressão intracraniana (PIC), $\mathrm{PtO}_{2}$ (pressão de oxigenação tecidual) e Doppler transcraniano vem com a proposta de monitorização longitudinal dentro de um continuum metabólico da isquemia cerebral. Em tese, esse tipo de monitorização seria ideal para determinar qual o ponto crítico em que uma intervenção cirúrgica deve ser realizada antes que o paciente siga o curso maligno do infarto cerebral. Entretanto, faz-se a ressalva de que a monitorização com microdiálise e $\mathrm{PtO}_{2}$ se restringe a uma pequena área do cérebro.

A perda da autorregulação é um fator-chave para determinar o curso maligno do infarto cerebral. Dohmen et al. ${ }^{7}$, utilizando neuromonitoramento da pressão de perfusão cerebral (PPC), $\mathrm{PtO}_{2}$ e microdiálise, mostraram relação significativa entre os índices de perda da autorregulação e a taxa lactato/piruvato com o desvio de linha média na TC e o Rankin após três meses. A partir dessa análise, conclui-se que os pacientes com perda da autorregulação devem ter PPC ajustados. A perda da autorregulação pode também ser aferida por meio dos índices do Doppler transcraniano. ${ }^{3,38}$

Quando a PPC cai abaixo do limiar crítico (50 a 60 $\mathrm{mmHg}$ ), o $\mathrm{PtO}_{2}$ também cai abaixo do seu limiar crítico $(10 \mathrm{mmHg})$ traduzindo uma hipóxia cerebral. Nesse momento, um aumento da relação lactato/piruvato e hipoxantina é detectado pela microdiálise, indicando que o metabolismo mudou de aeróbico para anaeróbico com aumento da quebra de ATP. Consequentemente, a concentração de aminoácidos excitatórios (glutamato, aspartato), glicerol e GABA também aumentaria. A literatura mostrou diferença estatisticamente significativa entre o curso maligno e benigno dos pacientes com relação às substâncias medidas pela microdiálise. Entretanto, essa diferença de curso só foi visível posteriormente aos sinais de herniação cerebral, quando essas substâncias atingiram seus valores de pico. ${ }^{8}$

A maioria dos tratamentos instituídos para edema cerebral maligno baseou-se na percepção do aumento da PIC como causa principal da deterioração neurológica. Entretanto, a causa mais provável dessa deterioração sob o olhar fisiopatológico parece ser a herniação do tecido infartado pelo edema citotóxico que cria um gradiente compartimental de pressões mais do que o aumento da PIC. ${ }^{9,16}$ Demonstrou-se ainda que o monitoramento da PIC não foi útil como parâmetro para o tratamento antiedema. Schwab et al..$^{41}$, num estudo sobre o valor da PIC no infarto maligno, mostraram que todos os pacientes do estudo apresentaram sinais de herniação antes da elevação da PIC. O mesmo estudo apresentou uma correlação significativa ( $<<0,0001$; $t$ test) dos valores máximos da PIC com o desfecho maligno do infarto cerebral. Conclui-se que o monitoramento da PIC não é um bom parâmetro para indicar a craniectomia descompressiva, pois os pacientes com infarto maligno herniam antes do aumento da PIC, atrasando uma possível intervenção. Além do mais, o estudo demonstrou que nenhum paciente com PIC > $35 \mathrm{mmHg}$ sobreviveu, e esse é um dado prognóstico importante com relação à contraindicação de cirurgia.

Burghaus et al. ${ }^{6}$ monitoraram 30 pacientes com infarto cerebral hemisférico dentro de 24 horas por meio do BAEP (Brainstem auditory evoked potential), objetivando determinar quais pacientes teriam curso maligno ou benigno, e encontraram BEAP patológico nos pacientes com curso maligno com diferença estatística $(\mathrm{p}<0,05)$.

\section{Tratamento}

Várias estratégias medicamentosas foram propostas para o infarto cerebral hemisférico, entretanto, nenhuma das medidas conservadoras foi eficaz nos estudos experimentais ou clínicos, e algumas medidas podem até mesmo acrescentar risco ao paciente. ${ }^{33,43}$ Apesar de alguns estudos apresentarem algum benefício, ${ }^{29}$ até o momento não há nenhuma recomendação baseada em evidência do uso de algum tipo de tratamento conservador., ${ }^{2,1744}$ A mortalidade do infarto cerebral hemisférico pode passar de $80 \%$, mesmo com tratamento conservador. ${ }^{14}$

A partir dos meados do século passado, houve um aumento no número de publicações sobre a craniectomia descompressiva para tratamento do infarto cerebral hemisférico. A maioria das publicações foram pequenos relatos de casos ou série de casos, ${ }^{24,11,18,21,32}$ que já demonstravam algum benefício com relação à mortalidade, entretanto, sem evidência científica suficiente. Os resultados dos três trials europeus (HAMLET, DESTINY, DECIMAL) reportados por Vahedi et al. ${ }^{45}$ finalmente vieram definir o nível de evidência que faltava para a indicação da craniectomia descompressiva para o infarto cerebral hemisférico em pacientes $<60$ anos com ictus $<48$ horas. A craniectomia descompressiva reduz mortalidade de $78 \%$ para $29 \%$ com NNT = 2 e melhora o seguimento funcional dos pacientes. Embora a chance de sobrevida com escala de Rankim modificada (mRS) 3 tenha dobrado, a sobrevida com mRS 4 aumentou 10 vezes. Esse aspecto deve ser discutido com a família antes de se indicar a cirurgia.

Com relação à técnica, a literatura relata ainda dois procedimentos complementares à craniectomia descompressiva convencional (craniectomia/duroplastia): a uncopara-hipocampectomia e strokecto- 
mia. ${ }^{22,31,39,44,48}$ Alguns autores sugerem que, às vezes, apenas a craniectomia com duroplastia seja insuficiente para descomprimir as estruturas mesiais já herniadas e propõem a ressecção cirúrgica direta do uncus e giro para-hipocampal (uncopara-hipocampectomia) ${ }^{31}$. Robertson et al. ${ }^{39}$ relataram 12 pacientes que fizeram, além da craniectomia descompressiva/duroplastia, a ressecção do parênquima infartado (strokectomia). Apesar de reduzir a taxa de mortalidade para $16 \%$ nesta série, a prevalência de sequelas graves foi maior do que as séries na literatura. Saber diferenciar macroscopicamente os limites entre área infartada, área de penumbra e área sadia é impossível no infarto cerebral agudo e por isso muitos autores questionam e não recomendam a strokectomia. ${ }^{11,18,44,48}$

Uma vez indicada a craniectomia descompressiva, ela deve ser generosa. $\mathrm{O}$ volume do hemisfério isquêmico pode aumentar até $50 \%$, dessa forma, o racional que se utiliza para a confecção da duroplastia é que a superfície de contato da área expandida tenha no mínimo $50 \%$ da área infartada. A craniectomia deve ter por volta de $10-12 \mathrm{~cm}$ anteroposteriormente e $14-15 \mathrm{~cm}$ de diâmetro da base temporal ao seio sargital. ${ }^{44}$

A hipotermia é outro tipo de tratamento que vem ganhando atenção na literatura para o tratamento do infarto cerebral hemisférico. $\mathrm{O}$ racional do efeito da hipotermia é que ela apresenta múltiplos e sinérgicos efeitos neuroprotetores, reduzindo a taxa de metabolismo cerebral, estabilizando a barreira hematoencefálica, reduzindo o edema cerebral, a formação de radicais livres e aminoácidos excitatórios e, finalmente, reduzindo a resposta inflamatória pós-isquêmica e apoptose. Entretanto, Georgiadis et al. ${ }^{10}$, comparando craniectomia descompressiva com hipotermia, concluíram que os resultados da hipotermia foram inferiores ao da craniectomia descompressiva.

Schwab et al. ${ }^{42}$ publicaram em 1998 o primeiro ensaio clínico sobre hipotermia e encontraram uma sobrevida de $56 \%$ dos pacientes que fizeram hipotermia moderada $\left(33^{\circ} \mathrm{C}\right)$ por 72 horas quando comparados à história natural da doença. Dessa forma, a hipotermia pode ser considerada um tratamento de segunda linha ou uma alternativa quando a craniectomia descompressiva estiver contraindicada, como no caso de cardiopatia severa, coagulopatias não reversíveis com transfusão, doença terminal, infarto bilateral de ACA ou MCA e condição funcional previamente ruim (mRS 4).

Vários autores têm recomendado a craniectomia descompressiva associada à hipotermia para maximinizar os resultados tanto da mortalidade quando da recuperação funcional que devem ser iniciados mesmo antes dos sinais de herniação. ${ }^{44}$ Entretanto, ainda faltam ensaios clínicos combinando as duas técnicas para mostrar esse potencial benefício adicional da hipotermia.

\section{Conclusões}

Não há recomendação baseada em evidência com bons resultados do uso do tratamento conservador, sendo a craniectomia descompressiva a primeira escolha para tratamento do infarto hemisférico maligno com nível de evidência 1 , para pacientes $<60$ anos dentro das primeiras 48 horas do ictus. Sua indicação precoce, ou seja, antes de sinais de herniação ou piora neurológica, pode ser baseada nos fatores preditivos significativos de curso maligno do infarto hemisférico.

A uncopara-hipocampectomia pode ser um procedimento complementar à craniectomia descompressiva quando o paciente já se encontra herniado. Nesse caso, a contraindicação de tratamento cirúrgico pode ser baseada nos valores da PIC ou da microdiálise, uma vez que valores de PIC > $35 \mathrm{mmHg}$ e valores de pico de aminoácidos excitatórios estão associados com desfecho letal em todos os casos.

A hipotermia moderada pode ser uma alternativa quando a craniectomia descompressiva estiver contraindicada, entretanto, seus resultados são inferiores ao tratamento cirúrgico.

\section{Agradecimentos}

Agradeço as orientações prestadas à conclusão desta minha monografia pelo professor Dr. Fabio Santana Machado, coordenador do curso de Pós-graduação em Neurointensivismo do Hospital Sírio-Libanês, São Paulo, SP.

\section{Referências}

1. Barber PA, Davis SM, Darby DG, Desmond PM, Gerraty RP, Yang $Q$, et al. Absent middle cerebral artery flow predicts the presence and evolution of the ischemic penumbra. Neurology. 1999;52(6):1125-32.

2. Bardutzky J, Schwab S. Antiedema therapy in ischemic stroke. Stroke. 2007;38(11):3084-94.

3. Bellapart J, Fraser JF. Transcranial Doppler assessment of cerebral autoregulation. Ultrasound Med Biol. 2009;35(6):883-93.

4. Berrouschot J, Rössler A, Köster J, Schneider D. Mechanical ventilation in patients with hemispheric ischemic stroke. Crit Care Med. 2000;28(8):2956-61.

5. Bozzao L, Fantozzi LM, Bastianello S, Bozzao A, Fieschi C. Early collateral blood supply and late parenchymal brain damage in patients with middle cerebral artery occlusion. Stroke. 1989;20(6):735-40.

6. Burghaus L, Liu WC, Dohmen C, Bosche B, Haupt WF. Evoked potentials in acute ischemic stroke within the first 
$24 \mathrm{~h}$ : possible predictor of a malignant course. Neurocrit Care. 2008;9(1):13-6.

7. Dohmen C, Bosche B, Graf R, Reithmeier T, Ernestus RI, Brinker $\mathrm{G}$, et al. Identification and clinical impact of impaired cerebrovascular autoregulation in patients with malignant middle cerebral artery infarction. Stroke. 2007;38(1):56-61.

8. Dohmen C, Bosche B, Graf R, Staub F, Kracht L, Sobesky $J$, et al. Prediction of malignant course in MCA infarction by PET and microdialysis. Stroke. 2003;34(9):2152-8.

9. Frank Jl. Large hemispheric infarction, deterioration, and intracranial pressure. Neurology. 1995;45(7):1286-90.

10. Georgiadis D, Schwarz S, Aschoff A, Schwab S. Hemicraniectomy and moderate hypothermia in patients with severe ischemic stroke. Stroke. 2002;33(6):1584-8.

11. Greenwood J Jr. Acute brain infarctions with high intracranial pressure: surgical indications. Johns Hopkins Med J. 1968;122(5):254-60.

12. Gupta R, Connolly ES, Mayer S, Elkind MS. Hemicraniectomy for massive middle cerebral artery territory infarction: a systematic review. Stroke. 2004;35(2):539-43.

13. Hacke W, Kaste M, Fieschi C, Toni D, Lesaffre E, von Kummer R, et al. Intravenous thrombolysis with recombinant tissue plasminogen activator for acute hemispheric stroke. The European Cooperative Acute Stroke Study (ECASS). JAMA. 1995;274(13):1017-25.

14. Hacke W, Schwab S, Horn M, Spranger M, De Georgia M, von Kummer R. 'Malignant' middle cerebral artery territory infarction: clinical course and prognostic signs. Arch Neurol. 1996;53(4):309-15.

15. Haring HP, Dilitz E, Pallua A, Hessenberger G, Kampfl A, Pfausler B, et al. Attenuated corticomedullary contrast: an early cerebral computed tomography sign indicating malignant middle cerebral artery infarction. A case-control study. Stroke. 1999;30(5):1076-82.

16. Hofmeijer J, Amelink GJ, Algra A, van Gijn J, Macleod MR, Kappelle LJ, et al. HAMLET investigators. Hemicraniectomy after middle cerebral artery infarction with life-threatening Edema trial (HAMLET). Protocol for a randomised controlled trial of decompressive surgery in space-occupying hemispheric infarction. Trials. 2006;7:29.

17. Hofmeijer J, van der Worp HB, Kappelle LJ. Treatment of space-occupying cerebral infarction. Crit Care Med. 2003;31(2):617-25.

18. Ivamoto HS, Numoto M, Donaghy RM. Surgical decompression for cerebral and cerebellar infarcts. Stroke. 1974;5(3):365-70.

19. Jaramillo A, Góngora-Rivera F, Labreuche J, Hauw JJ, Amarenco P. Predictors for malignant middle cerebral artery infarctions: a postmortem analysis. Neurology. 2006;66(6):815-20.

20. Jüttler $E$, Schwab S, Schmiedek $P$, Unterberg A, Hennerici M, Woitzik J, et al. DESTINY Study Group. Decompressive Surgery for the Treatment of Malignant Infarction of the Middle Cerebral Artery (DESTINY): a randomized, controlled trial. Stroke. 2007;38(9):2518-25.

21. Kakita K, Miyazaki T, Kadowaki H, Izawa M, Kubota S. [A trial of surgical management of brain edema in cerebral infarction--a review with our own experiences in 31 cases (author's transl)]. No Shinkei Geka. 1976;4(3):277-83.

22. Kalia KK, Yonas H. An aggressive approach to massive middle cerebral artery infarction. Arch Neurol. 1993;50(12):1293-7.

23. Kasner SE, Demchuk AM, Berrouschot J, Schmutzhard E, Harms L, Verro P, et al. Predictors of fatal brain edema in massive hemispheric ischemic stroke. Stroke. 2001;32(9):2117-23.
24. King $A B$. Massive cerebral infarction producing ventriculographic changes suggesting a brain tumor. $J$ Neurosurg. 1951;8(5):536-9.

25. Krayenbuhl $\mathrm{H}$, Yasargil G. [Angiography of collateral cerebral circulation]. Acta Neurochir (Wien). 1958;6(1-2):30-80.

26. Krieger DW, Demchuk AM, Kasner SE, Jauss M, Hantson L. Early clinical and radiological predictors of fatal brain swelling in ischemic stroke. Stroke. 1999;30(2):287-92.

27. Lam WW, Leung TW, Chu WC, Yeung DT, Wong LK, Poon WS. Early computed tomography features in extensive middle cerebral artery territory infarct: prediction of survival. J Neurol Neurosurg Psychiatry. 2005;76(3):354-7.

28. Limburg M, van Royen EA, Hijdra A, de Bruïne JF, Verbeeten BW Jr. Single-photon emission computed tomography and early death in acute ischemic stroke. Stroke. 1990;21(8):1150-5.

29. Maramattom BV, Bahn MM, Wijdicks EF. Which patient fares worse after early deterioration due to swelling from hemispheric stroke? Neurology. 2004;63(11):2142-5.

30. Mori K, Aoki A, Yamamoto T, Horinaka N, Maeda M. Aggressive decompressive surgery in patients with massive hemispheric embolic cerebral infarction associated with severe brain swelling. Acta Neurochir (Wien). 2001;143(5):483-91.

31. Mori K, Ishimaru S, Maeda M. Unco-parahippocampectomy for direct surgical treatment of downward transtentorial herniation. Acta Neurochir (Wien). 1998;140(12):1239-44.

32. Mracek Z. [Significance of decompression craniotomy in acute occlusion of the middle cerebral artery with brain-stem symptomatology due to pressure by the edematous cerebral hemisphere]. Cesk Neurol Neurochir. 1978;41(6):390-3.

33. Muizelaar JP, Marmarou A, Ward JD, Kontos HA, Choi SC, Becker DP, et al. Adverse effects of prolonged hyperventilation in patients with severe head injury: a randomized clinical trial. J Neurosurg. 1991;75(5):731-9.

34. Murray CJ, Lopez AD. Mortality by cause for eight regions of the world: Global Burden of Disease Study. Lancet. 1997;349(9061):1269-76.

35. Neumann-Haefelin T, Moseley ME, Albers GW. New magnetic resonance imaging methods for cerebrovascular disease: emerging clinical applications. Ann Neurol. 2000;47(5):559-70.

36. $\mathrm{Ng}$ LK, Nimmannitya J. Massive cerebral infarction with severe brain swelling: a clinicopathological study. Stroke. 1970;1(3):158-63.

37. Oppenheim C, Samson Y, Manaï R, Lalam T, Vandamme $\mathrm{X}$, Crozier S, et al. Prediction of malignant middle cerebral artery infarction by diffusion-weighted imaging. Stroke. 2000;31(9):2175-81.

38. Panerai RB. Transcranial Doppler for evaluation of cerebral autoregulation. Clin Auton Res. 2009;19(4):197-211.

39. Robertson SC, Lennarson P, Hasan DM, Traynelis VC. Clinical course and surgical management of massive cerebral infarction. Neurosurgery. 2004;55(1):55-61.

40. Saito I, Segawa H, Shiokawa Y, Taniguchi M, Tsutsumi K. Middle cerebral artery occlusion: correlation of computed tomography and angiography with clinical outcome. Stroke. 1987;18(5):863-8.

41. Schwab S, Aschoff A, Spranger M, Albert F, Hacke W. The value of intracranial pressure monitoring in acute hemispheric stroke. Neurology. 1996;47(2):393-8.

42. Schwab S, Schwarz S, Spranger M, Keller E, Bertram $M$, Hacke W. Moderate hypothermia in the treatment of patients with severe middle cerebral artery infarction. Stroke. 1998;29(12):2461-6. 
43. Schwab S, Spranger M, Schwarz S, Hacke W. Barbiturate coma in severe hemispheric stroke: useful or obsolete? Neurology. 1997;48(6):1608-13.

44. Steiner T, Ringleb P, Hacke W. Treatment options for large hemispheric stroke. Neurology. 2001;57(5 Suppl 2):S61-8.

45. Vahedi K, Hofmeijer J, Juettler E, Vicaut E, George B, Algra A, et al. DECIMAL, DESTINY, and HAMLET investigators. Early decompressive surgery in malignant infarction of the middle cerebral artery: a pooled analysis of three randomised controlled trials. Lancet Neurol. 2007;6(3):215-22.

46. Vahedi K, Vicaut E, Mateo J, Kurtz A, Orabi M, Guichard JP, et al. DECIMAL Investigators. Sequential-design, multicenter, randomized, controlled trial of early decompressive craniectomy in malignant middle cerebral artery infarction (DECIMAL Trial). Stroke. 2007;38(9):2506-17.

47. von Kummer R, Meyding-Lamadé $U$, Forsting M, Rosin L, Rieke K, Hacke W, et al. Sensitivity and prognostic value of early CT in occlusion of the middle cerebral artery trunk. AJNR Am J Neuroradiol. 1994;15(1):9-15.
48. Wijdicks EF. Management of massive hemispheric cerebral infarct: is there a ray of hope? Mayo Clin Proc. 2000;75(9):945-52.

49. Yao Y, Liu W, Yang X, Hu W, Li G. Is decompressive craniectomy for malignant middle cerebral artery territory infarction of any benefit for elderly patients? Surg Neurol. 2005;64(2):165-9.

\section{Endereço para correspondência}

Wuilker Knoner Campos

Instituto de Neurocirurgia Neuron, sala 419,

Baia Sul Medical Center

Rua Menino Deus, 63

88020-210 - Florianópolis, SC

Tel.: (48) 3224-0843

Email: wuilker@yahoo.com.br 